\title{
Imaging of Flow Patterns with Fluorescent Molecular Rotors
}

\author{
Adnan Mustafic • Hsuan-Ming Huang • \\ Emmanuel A. Theodorakis • Mark A. Haidekker
}

Received: 3 February 2010 / Accepted: 29 March 2010 / Published online: 20 April 2010

(C) The Author(s) 2010. This article is published with open access at Springerlink.com

\begin{abstract}
Molecular rotors are a group of fluorescent molecules that form twisted intramolecular charge transfer states (TICT) upon photoexcitation. Some classes of molecular rotors, among them those that are built on the benzylidene malononitrile motif, return to the ground state either by nonradiative intramolecular rotation or by fluorescence emission. In low-viscosity solvents, intramolecular rotation dominates, and the fluorescence quantum yield is low. Higher solvent viscosities reduce the intramolecular rotation rate, thus increasing the quantum yield. We recently described a different mechanism whereby the fluorescence quantum yield of the molecular rotor also depends on the shear stress of the solvent. In this study, we examined a possible application for shear-sensitive molecular rotors for imaging flow patterns in fluidic chambers. Flow chambers with different geometries were constructed from polycarbonate or acrylic. Solutions of molecular rotors in ethylene glycol were injected into the chamber under controlled flow rates. LED-induced fluorescence
\end{abstract}

\footnotetext{
A. Mustafic · M. A. Haidekker $(\varangle)$

Faculty of Engineering, Driftmier Engineering Center, University of Georgia, Athens, GA 30602, USA

e-mail:mhaidekk@uga.edu

H.-M. Huang

Department of Biomedical Engineering,

Case Western Reserve University,

Cleveland, OH 44106, USA

\section{E. A. Theodorakis}

Department of Chemistry and Biochemistry,

University of California, San Diego, 9500 Gilman Drive,

San Diego, CA 92093, USA
}

(LIF) images of the flow chambers were taken with a digital camera, and the intensity difference between flow and no-flow images was visualized and compared to computed fluid dynamics (CFD) simulations. Intensity differences were detectable with average flow rates as low as $0.1 \mathrm{~mm} / \mathrm{s}$, and an exponential association between flow rate and intensity increase was found. Furthermore, a good qualitative match to computed fluid dynamics simulations was seen. On the other hand, prolonged exposure to light reduced the emission intensity. With its high sensitivity and high spatial and temporal resolution, imaging of flow patterns with molecular rotors may become a useful tool in microfluidics, flow measurement, and control.

Keywords Molecular rotors • Flow sensors • Shear stress $\cdot$ Twisted intramolecular charge transfer states (TICT) • Microfluidics • Computed fluid dynamics

\section{Introduction}

Molecular rotors are a group of fluorescent molecules with known applications as non-mechanical viscosity sensors with high spatial and temporal resolution [1-4]. This unique sensitivity to the microviscosity of the environment has established molecular rotors as a promising alternative to mechanical viscometers $[5,6]$.

The chemical structure of the molecular rotors contains an electron donor unit, such as the nitrogen atom, in conjugation with an electron acceptor unit, such as the nitrile unit. Intramolecular rotation occurs around the $\sigma$-bonds that connect the electronically rich 
$\pi$-system with the donor and acceptor groups (Fig. 1). Notably, when the molecule assumes a twisted conformation, the energy difference between excited state and ground state is low enough to cause radiationless deexcitation [7]. Therefore, only deexcitation from the planar (LE) state leads to fluorescence emission. Unlike other molecular rotors, such as 4,4dimethylaminobenzonitrile (DMABN), the molecules shown in Fig. 1 exhibit a single fluorescence emission band. In solutions with low viscosity, the intramolecular rotation is the predominant deexcitation pathway, whereas in high-viscosity solutions, the intramolecular rotation is impeded, and fluorescence emission increases as the intramolecular rotation rate decreases $[3,8]$. The fluorescence quantum yield depends on the viscosity of the environment in a power-law fashion as first described by Förster and Hoffmann [9],

$\log \phi_{F}=x \log \eta+C$

where $\phi_{F}$ is fluorescence quantum yield, $\eta$ is a solvent's viscosity, and $C$ and $x$ are dye-and solvent-dependent constants [10].

The two molecular rotors examined in this study are shown in Fig. 1. The first molecule is 9-(2-carboxy2-cyanovinyl)-julolidine (abbreviated CCVJ), which is soluble in many polar solvents, such as alcohols and water. The carboxylic acid functionality can be used to form esters, among them the water-soluble CCVJtri ethylene glycol [11] and p-[(2-cyano-2-propanediol ester) vinyl] dimethylaniline (abbreviated CPVDA). When these polar molecular rotors are dissolved in polar solvents, increased fluorescence intensity can be observed when the solution is exposed to shear stress [12]. This effect allows to envision molecular rotors as
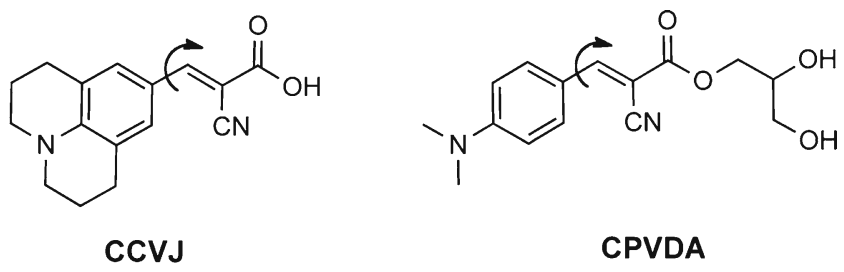

Fig. 1 Chemical structures of the molecular rotors CCVJ (9(2-carboxy-2-cyanovinyl)-julodine) and CPVDA (p-[(2-cyano-2propanediol ester) vinyl] dimethylaniline). Excited-state charge transfer takes place from the julolidine- or dimethylaminonitrogen atom to the nitrile group. In the excited state, the molecule tends to rotate the 2-cyanoprop-2-enoic acid group relative to the aniline or julolidine group (formation of the twisted intramolecular charge transfer state). Arrows denote the axis of rotation tools to measure flow and to image flow patterns. In this study, we demonstrate how images of the velocitydependent fluorescence intensity can be obtained, and we relate these images to flow velocity, chamber geometry, and to flow patterns simulated with computed fluid dynamics (CFD).

\section{Materials and methods}

\section{Basic shear sensitivity experiment}

A solution of $10-\mu \mathrm{M}$ CCVJ (Sigma, St. Louis, MO) and CPVDA (synthesized by our group) in fluorescence grade ethylene glycol (Sigma, St. Louis, MO) was placed in a fluoroscopic glass cuvette, and a magnetic stir bar was introduced. In a flurophotometer (Fluoromax 3, Jobin-Yvon, Edison, NJ), emission spectra from $450 \mathrm{~nm}$ to $550 \mathrm{~nm}$ were acquired with an excitation wavelength of $422 \mathrm{~nm}$ for CCVJ and $434 \mathrm{~nm}$ for CPVDA. Spectra with the stirrer turned off (no shear) and with the stirrer at a low setting (moderate shear without turbulence or vortex formation) were acquired and compared.

\section{Chamber construction}

Flow chambers (the geometries shown in Fig. 2) used in this study were constructed from Delrin and polycarbonate. Dimensions were $84 \times 44 \mathrm{~mm}$. Flow channels with a depth of $3 \mathrm{~mm}$ were milled into the polymer, and the chamber closed with a borosilicate microscope glass slide (Corning Inc., Corning, NY) that was glued onto the polymer block. The flow chamber was then placed on top of two posts in front of the emission filter and the LED excitation sources. The imaging apparatus consisted of a conventional SLR Camera (Konica Minolta Dynax Maxxum 7D equipped with a Sigma $50 \mathrm{~mm} \mathrm{f} / 2.8$ fixed-focus lens), a D450/40 image grade emission bandpass filter (Chroma Technology Corp, Brattleboro, VT) that was placed directly in front of the lens, two high-power LED excitation sources (Philips/Luxeon LXHL-LR5C) with added excitation filters to reduce the spectral bandwidth of the emission, two posts for the flow chamber, and one post each for the SLR Camera and emission filter. The LED sources provided broad illumination to cover the entire front of a flow chamber. LED excitation sources were connected to a custom LED driver, and provided either continuous homogeneous light or intensity-modulated light. 
Fig. 2 Sketch of the chamber geometries examined in this study. A simple hexagonal chamber (a) was used to feature a relatively large section of homogeneous flow. The cleaver-shaped chamber (b) provides a narrow entry channel with high fluid velocities and a larger basin with low average velocities and two stagnant regions. In two more chambers flow is routed around an island. In c, two narrow sections of high flow velocity above and below the island exist, whereas in $\mathbf{d}$, the channel width is kept constant, so that the flow is divided into two streams above and below the island that each have half off the velocity of the inlet channel
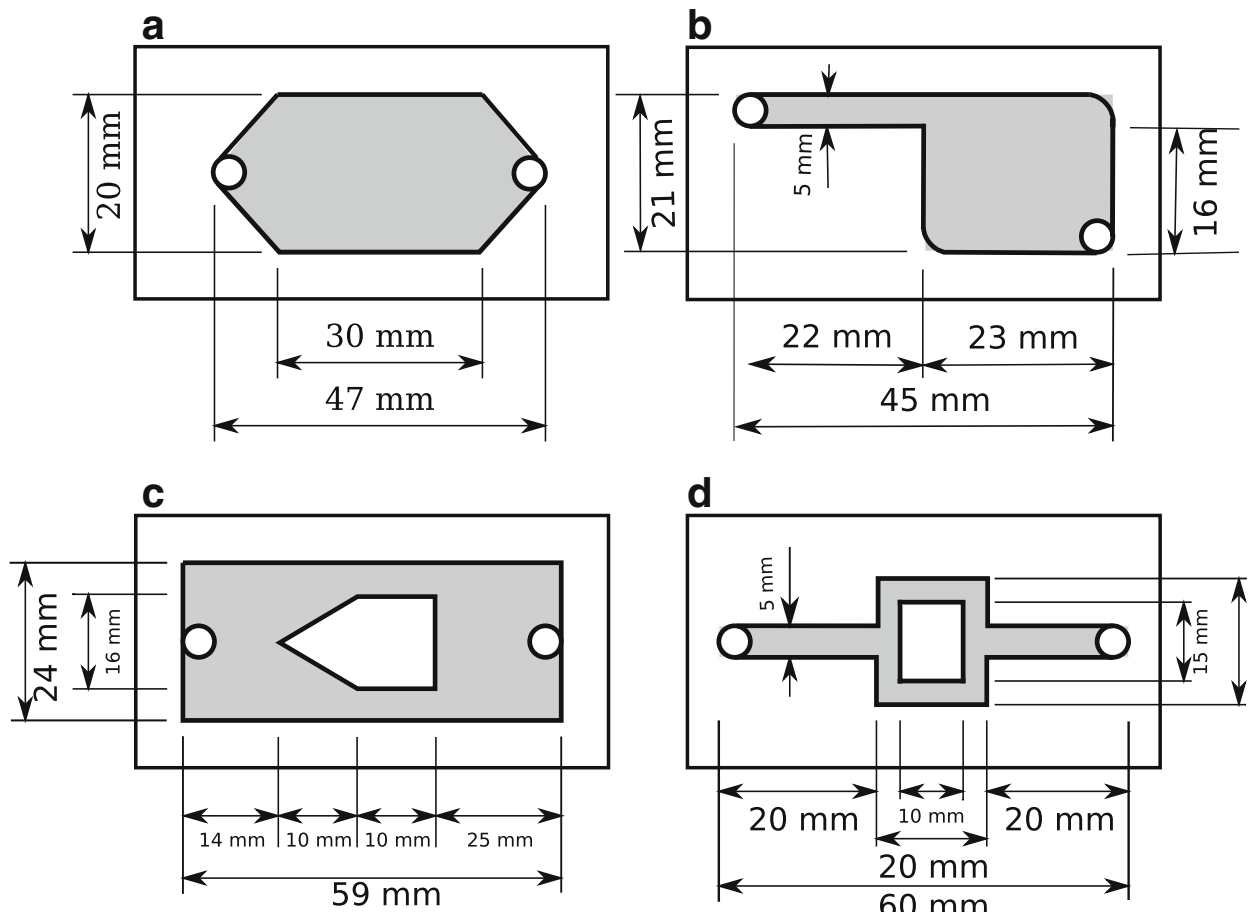

d

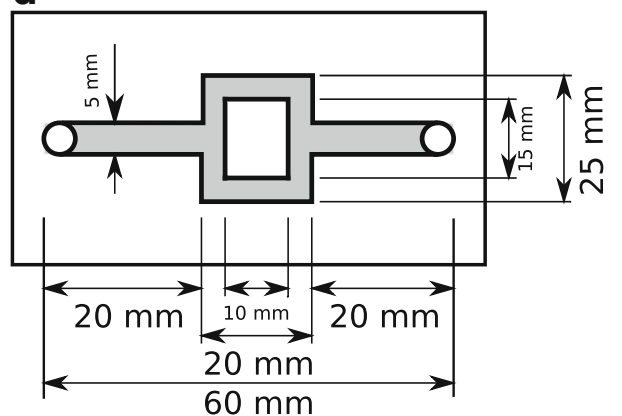

Particle tracking with polystyrene microspheres in ethylene glycol

Fluorescent polystyrene microspheres for flow determination (Molecular Probes, Carlsbad, CA) were used to make a $14-\mu \mathrm{M}$ solution in $25 \mathrm{ml}$ of ethylene glycol. The green fluorescent microspheres measured $15 \mu \mathrm{M}$ in diameter with absorption and emission wavelengths at $450 \mathrm{~nm}$ and $480 \mathrm{~nm}$, respectively. A concentration of $1.0 \times 10^{6}$ beads $/ \mathrm{ml}$ was used. Camera settings included exposure at $8 \mathrm{~s}$ and $\mathrm{f} / 2.8$ aperture with an ISO setting of 800 . Because of the long exposure time, the brightly fluorescent beads showed up as streaks in the image, representing the streamlines.

Preparing and injecting a viscous molecular rotor solution

A solution was made from $60 \mu \mathrm{l}$ of $10 \mathrm{mM}$ molecular rotor stock solution in $40 \mathrm{ml}$ of fluorescence-grade ethylene glycol, a solvent with viscosity of approximately $14 \mathrm{mPa} \cdot \mathrm{s}$. The solution of a molecular rotor in ethylene glycol was thoroughly mixed and filled into a $30-\mathrm{ml}$ syringe. The syringe was then positioned in a custom syringe pump to provide precise and controllable flow rates. A short flexible polyethylene tube connected the syringe to the flow chamber inlet (connections estab- lished with Luer-Lok components). The chamber outlet was in turn connected to another syringe, positioned upright, which collected the excess fluid.

Image acquisition and processing

Images were acquired with an exposure time of $8 \mathrm{~s}$ and lens aperture of $\mathrm{f} / 2.8$. The camera sensitivity was set to ISO 200. The camera was set to acquire rawformat images to make use of the full 12 bit-per-pixel dynamic range. One set of images was taken each at $0.25,0.50,0.75,1.00,1.50 \mathrm{ml} / \mathrm{min}$ flow rate. For each flow rate, two images with flow and two images without flow (at rest) were taken in succession. No-flow images were intended for background intensity normalization. After each experiment, all images were transferred to a computer for image analysis.

Image processing tools were: (1) UFRaw, a conversion tool from the raw image format to 12 bit-per-pixel TIFF images, and (2) Crystal Image (quantitative image analysis software) [13], all software running on Ubuntu Linux version 8.04. In preparation for image processing, the blue channel with peak sensitivity wavelength of $450 \mathrm{~nm}$ was extracted from the color image file and stored as a single-channel grayscale image for the following processing and analysis steps. These consisted of: (1) a median filter applied to each image individually 
to reduce noise, (2) reduction of the image size from $3,008 \times 2,000$ pixels to $752 \times 500$ pixels by binning, and (3) computation of a normalized difference image by using the following equation embedded in a Crystal Image macro,

$$
\begin{aligned}
& I(x, y) \\
& =\frac{\left(I_{F 1}(x, y)+I_{F 2}(x, y)\right)-\left(I_{N F 1}(x, y)+I_{N F 2}(x, y)\right)}{I_{N F 1}(x, y)+I_{N F 2}(x, y)}
\end{aligned}
$$

where $I_{F 1}(x, y)$ and $I_{F 2}(x, y)$ represent the two flow images, and $I_{N F 1}(x, y)$ and $I_{N F 2}(x, y)$ represent the two no-flow images. By creating the normalized difference images, any background inhomogeneities were removed. By using two images added together, signal-tonoise ratio was increased by about $40 \%$. The resulting normalized image was further denoised with a Gaussian smoothing filter. The resulting image was false-colored to enhance visual perception of contrast. Where appropriate, average intensities were obtained by defining a rectangular region of interest and averaging all intensity values $I(x, y)$ as defined in Eq. 2 inside the region of interest. Any further processing of tabular data (namely, averaged $I(x, y)$ as a function of average flow rate and averaged $I(x, y)$ as a function of the horizontal coordinate) was performed with Graphpad Prism (version 4.01, Graphpad Inc., San Diego).

\section{Computed fluid dynamics}

For the computed fluid dynamics (CFD) modeling, the open-source software NaSt3DGP (developed by the research group of the Division of Scientific Computing and Numerical Simulation at the University of Bonn, Germany) was used [14]. NaSt3DGP allows the specification of arbitrary geometries. Flow chamber geometries were discretized at 360 by 180 by 80 points after it was determined that further refinement of the grid did not change the simulation results. Entry and exit ports were modeled as circular openings with constant-flow and zero-pressure boundary conditions, respectively. All other boundary cells were set to a no-slip boundary condition. After convergence, three matrices were obtained with the velocity components in $\mathrm{x}, \mathrm{y}$, and $\mathrm{z}$ direction. A scalar flow field was computed by using Eq. 3,

$$
|v|=\sqrt{v_{x}^{2}+v_{y}^{2}+v_{z}^{2}}
$$

and projected into two-dimensional space by averaging the velocity in all cells along the $\mathrm{z}$-axis. The resulting projected flow magnitude was visualized with the same method as the fluorescent images. CFD images were only used for a qualitative comparison to fluorescent flow pattern images.

\section{Results}

The basic experiment where both a CCVJ and CPVDA solution in ethylene glycol were stirred, demonstrates the fundamental finding of this study in a qualitative manner: when the solvent was in motion, fluorescence emission intensity was increased by about $15 \%$ for CCVJ and about $6 \%$ for CPVDA (Fig. 3). After cessation of shear stress (stopping of the stirrer) intensity dropped back to at-rest levels before the experiment. No spectral shift was observed in the fluid in motion.

Spatially resolved fluorescence intensity was acquired in four different chamber geometries. In all cases, overall intensity increased with flow rate. In addition, in narrower regions with expected higher flow rate, a higher intensity was observed. One image of the molecular rotor emission intensity is shown in Fig. 4. From top to bottom, flow rate was increased from $0.5 \mathrm{ml} / \mathrm{min}, 1.0 \mathrm{ml} / \mathrm{min}$ to $1.5 \mathrm{ml} / \mathrm{min}$. Additional images were taken at $0.25 \mathrm{ml} / \mathrm{min}$ and $0.75 \mathrm{ml} / \mathrm{min}$. These five flow rates translate into average fluid velocities of $0.07,0.14,0.21,0.28$, and $0.42 \mathrm{~mm} / \mathrm{s}$. At $1 \mathrm{ml} / \mathrm{min}$, a fluid particle would need $107 \mathrm{~s}$ to cross the rectangular part of the channel. Average intensity inside the rectangular section of the chamber was determined and plotted as a function of the distance from the inlet port (Fig. 5). From these curves, it can be seen that emission intensity increases with fluid velocity in a nonlinear fashion. At a flow rate of $0.25 \mathrm{ml} / \mathrm{min}$, fluorescence intensity is not noticeably different from background.
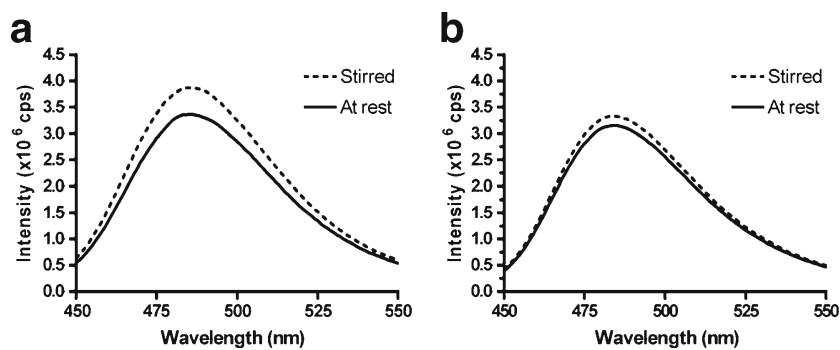

Fig. 3 Fluorescent emission spectra of $10 \mu \mathrm{M}$ CCVJ in ethylene glycol (a) and $10 \mu \mathrm{M}$ CPVDA in ethylene glycol (b) in a fluoroscopic cuvette with and without stirring. An increased emission intensity, but no spectral shift, can be observed when the stirrer is turned on to provide moderate fluid motion without turbulence or vortex formation 

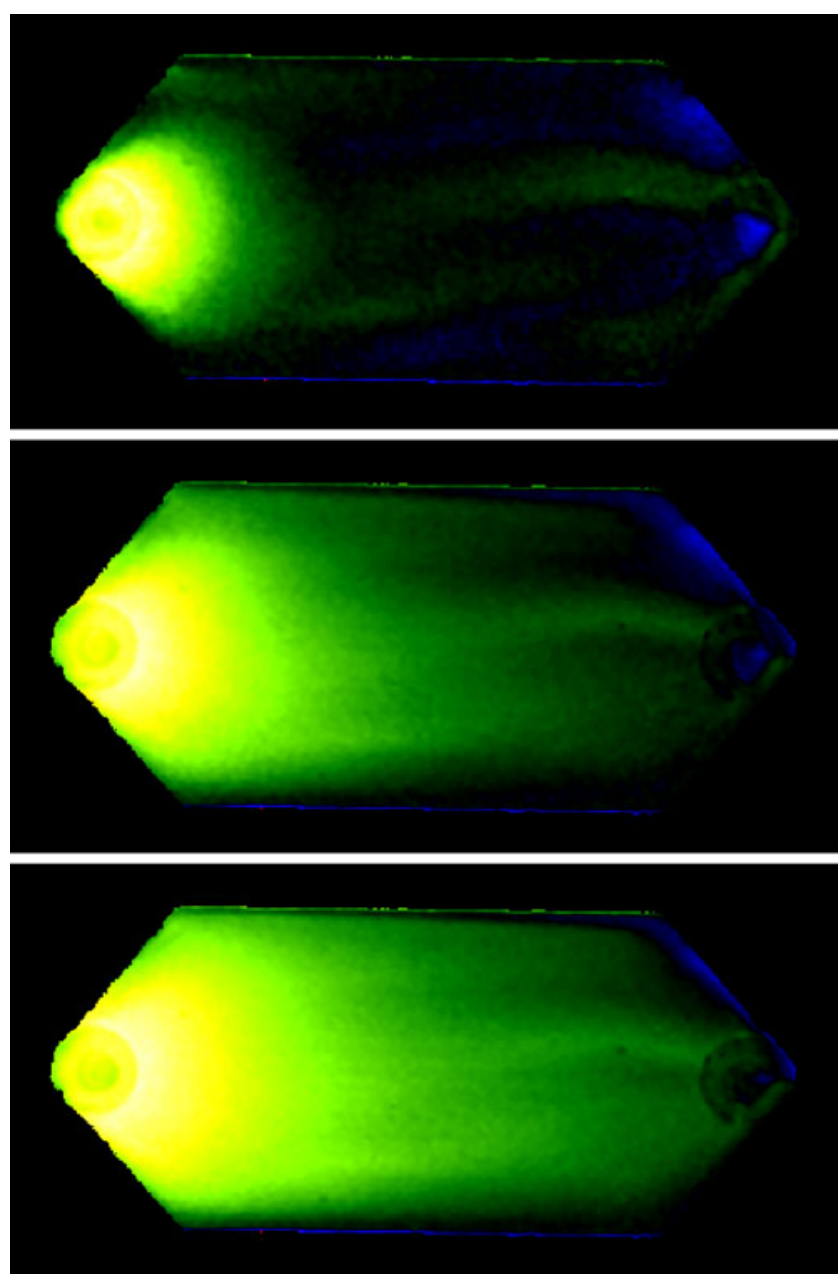

Fig. 4 Spatially resolved emission intensity (fluorescence image) of ethylene glycol with CCVJ flowing in the chamber shown in Fig. $2 \mathrm{a}$ at $0.5,1$, and $1.5 \mathrm{ml} / \mathrm{min}$ (top to bottom). Emission intensity increases visibly with flow rate, but seems to decrease over the length of the flow chamber

Between $0.5 \mathrm{ml} / \mathrm{min}$ and $1.0 \mathrm{ml} / \mathrm{min}$, intensity increases almost linearly, and at $1.5 \mathrm{ml} / \mathrm{min}$, a saturation effect can be observed. The nonlinear, dye-dependent relationship between fluorescence intensity and velocity is further highlighted in Fig. 6 with both dyes, CCVJ and CPVDA. Nonlinear curve fitting of the data in Fig. 6 with Eq. 4 was performed [12].

$\Delta I=\Delta I_{\max } \cdot\left(1-\exp \left(-\frac{v}{v_{c}}\right)\right)$

Here, $v$ is the fluid velocity and $\Delta I$ is the corresponding intensity above no-flow background. $\Delta I_{\max }$ and $v_{c}$ are constants obtained through nonlinear regression. The values obtained for $v_{c}$ in CCVJ were

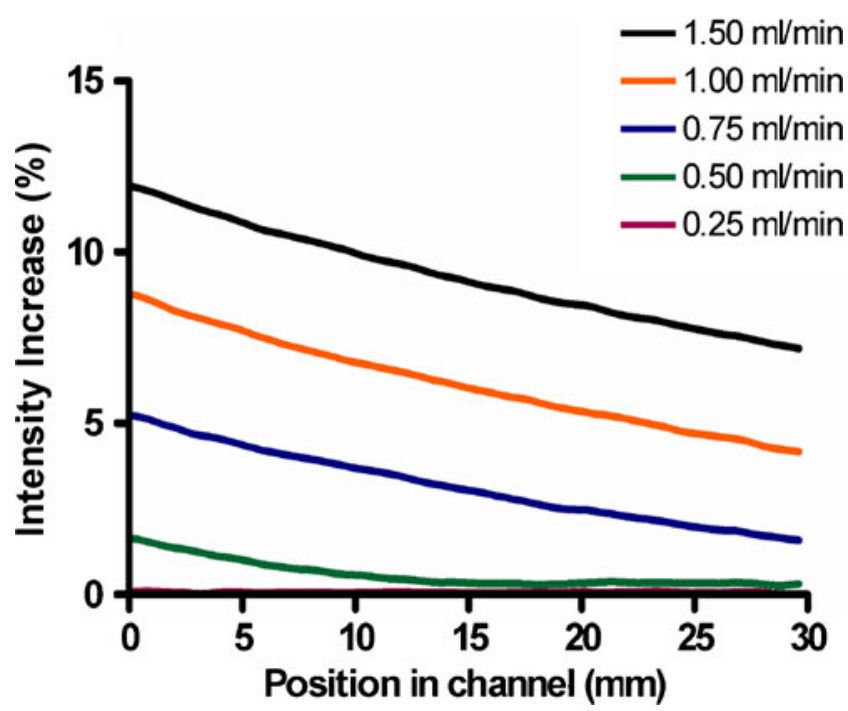

Fig. 5 Average fluorescence intensity $(\mathrm{CCVJ})$ in the central region of the hexagonal chamber geometry (Fig. 4) shown as a function of the distance from the left edge of the square section

$0.64 \mathrm{ml} / \mathrm{min}\left(\mathrm{R}^{2}=0.99\right)$, and for CPVDA $5.56 \mathrm{ml} / \mathrm{min}$ $\left(\mathrm{R}^{2}=0.94\right)$. The extrapolated maximum intensity increase was $\Delta I_{\max }=49 \%$ for CCVJ and $\Delta I_{\max }=25 \%$ for CPVDA. At a flow rate of $4 \mathrm{ml} / \mathrm{min}$, the intensity increase was approximately three times larger for CCVJ in ethylene glycol than for CPVDA in ethylene glycol, whereby CCVJ showed a stronger saturation effect than CPVDA at this flow rate. Higher flow rates than 4 $\mathrm{ml} / \mathrm{min}$ were not possible because of the pressure buildup in the chamber. CCVJ exhibits higher sensitivity towards flow, but saturates at lower flow rates than CPVDA.

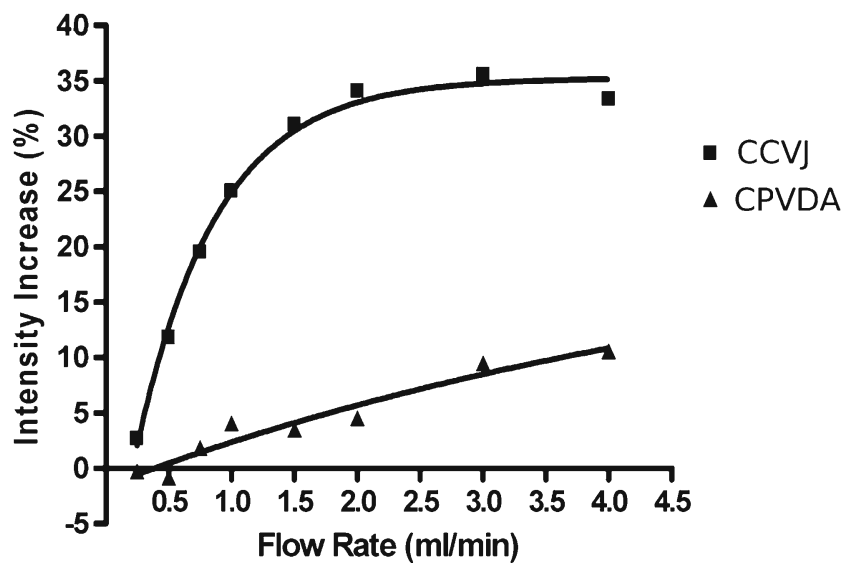

Fig. 6 Flow rate vs. emission intensity increase for CCVJ and CPVDA (both in ethylene glycol) in the hexagonal chamber geometry shown in Fig. 4. The nonlinear regression curves are described by Eq. 4 


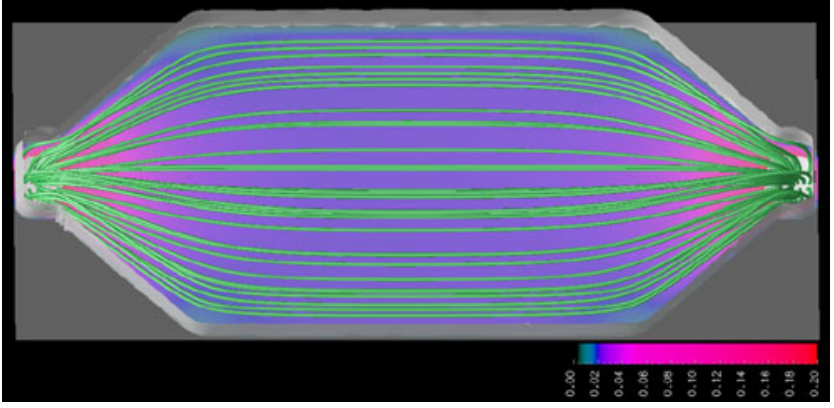

Fig. 7 Streamlines in the hexagonal chamber (Fig. 2a) simulated with computed fluid dynamics. Streamlines are shown in green superimposed over a false-colored map of the average velocity. The color bar indicates average flow velocity in millimeter per second for this particular simulation

Additionally, some fading of the fluorescence intensity over distance from left to right was observed (Fig. 5). To further examine the possible causes of the fading effect, streamlines were acquired by using fluorescent microbeads in pure ethylene glycol without any molecular rotor dye. CFD reveals a regular and symmetric flow pattern with corresponding symmetric streamlines (Fig. 7). Photographs of the fluorescent microbeads at rest and at $1.5 \mathrm{ml} / \mathrm{min}$ are shown in Fig. 8. Although some microbeads did not move, likely because they were stuck to the chamber surface, Fig. 8 corresponds closely with CFD, and no anomalies or irregularities in the downstream regions of the chamber could be observed. Further experiments were performed with strobed LED light and with partial occlusion of the chamber (data not shown).

For the flow chamber geometry shown in Fig. 2b, an excellent match with flow velocity, computed using CFD simulations, was found (Figs. 9 and 10). The higher intensity in the narrow inflow channel with its associated higher flow rate compared to the wide basin is particularly well visible. Similarly, the stagnant zones
Fig. 8 Fluorescent

microbeads at rest (top) and at $1.50 \mathrm{ml} / \mathrm{min}$ (bottom) in the hexagonal chamber geometry. The fluorescent microbeads in motion are represented by streaks in the image. Those streaks closely resemble the streamlines in Fig. 7 and show no irregularities or asymmetries in the chamber
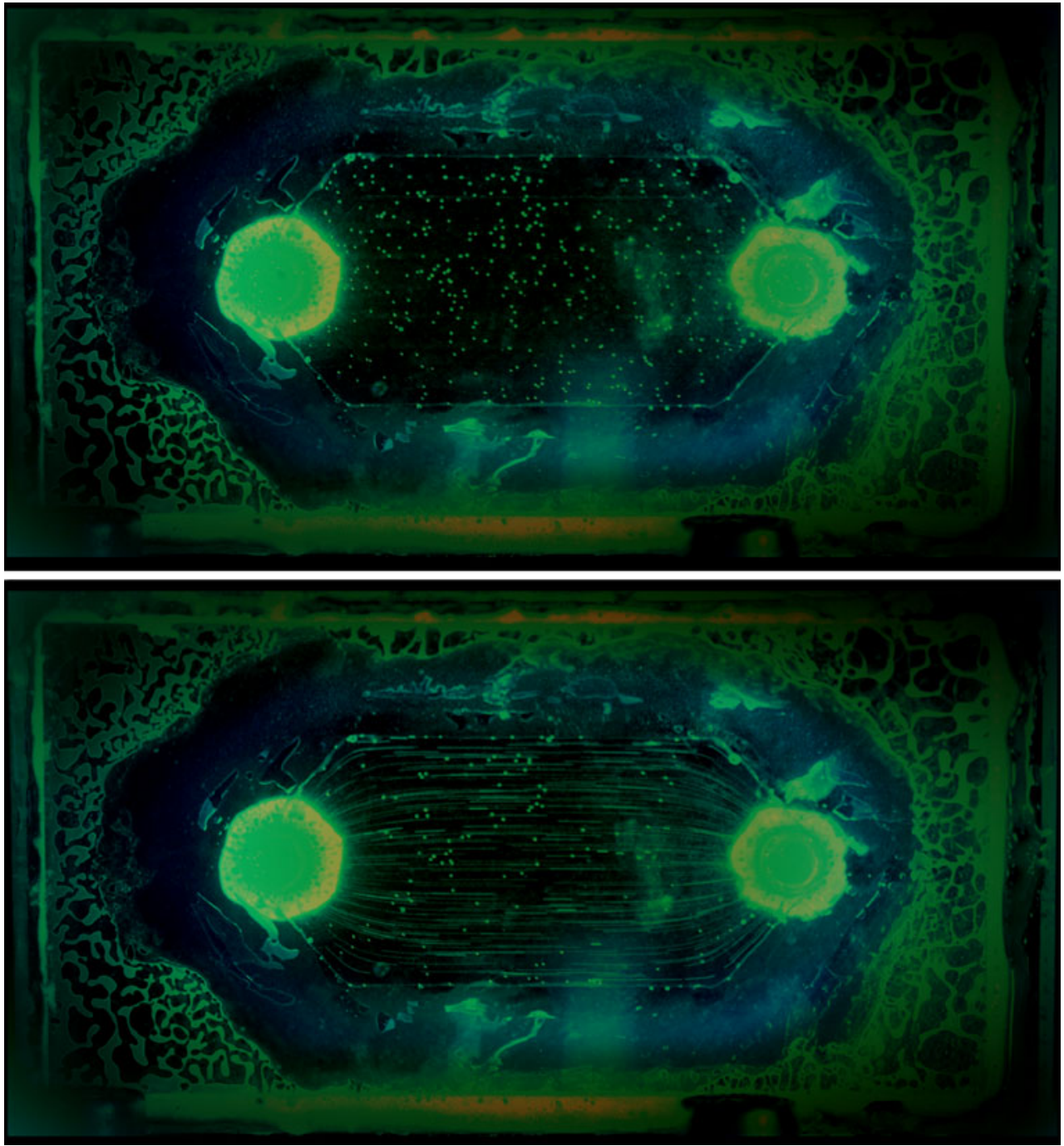

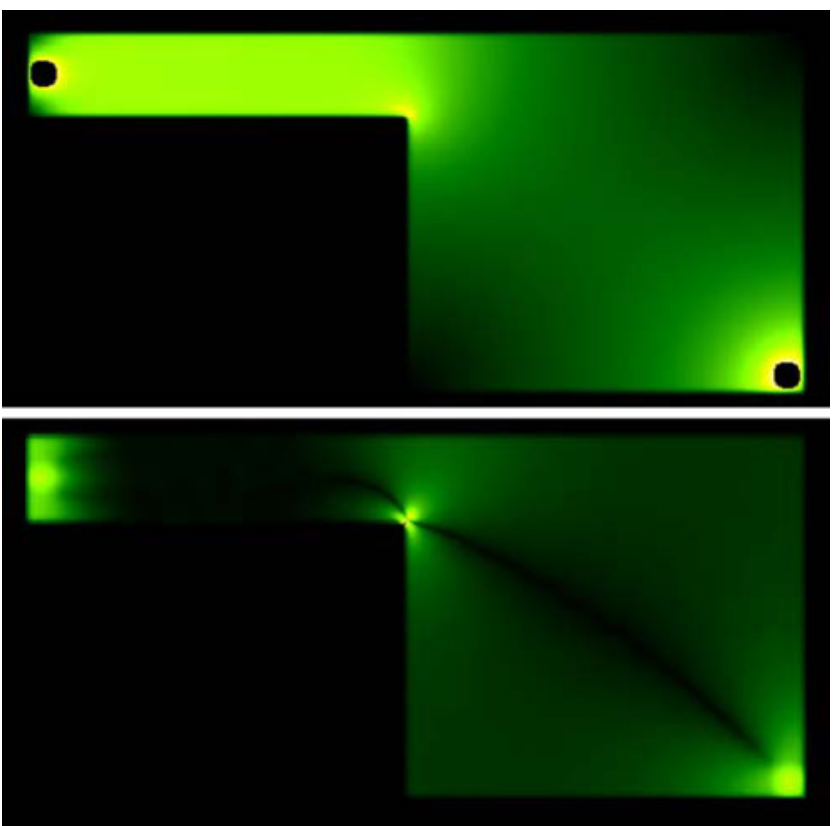

Fig. 9 Computed fluid dynamics simulation of the chamber depicted in Fig. $2 \mathrm{~b}$ at $1 \mathrm{ml} / \mathrm{min}$. Top image shows the projected fluid velocity, whereas the bottom image shows the projected fluid shear stress. There is a strong similarity between the velocity intensity pattern of the CFD simulation and the acquired fluorescence intensity in Fig. 10. The similarities extend to the region where the small channel enters the basin, and to the two stagnant areas in the top right and bottom left borders of the basin, where corresponding low fluorescence is visible in Fig. 10

in the top right and bottom left corners of the basin can easily be identified. On the other hand, the simulated pattern of shear stress (lower image in Fig. 9) shows a region of very homogeneous flow in the inflow channel with correspondingly low shear stress, and exhibits very characteristic symmetry lines. None of these can be found in the fluorescence image (Fig. 10).

With the third geometry (Fig. 11), the agreement between fluorescent images and CFD flow images (Fig. 12) is not as pronounced. However, the dark region at the symmetry axis in Fig. 11 is consistent with low shear stress that is expected where the two stream parts join. The fading effect that was already observed with the first geometry is particularly strong. In this geometry, a parabolic intensity profile can be observed particularly well in the narrow sections above and below the central island.

The fourth geometry shown in Fig. 2d shows an almost instantaneous drop in fluorescence intensity where the streams split into the upper and lower stream (Fig. 13). The channels above and below the rectangular island can be expected to have approximately one half of the flow rate of the inlet channel, an effect that
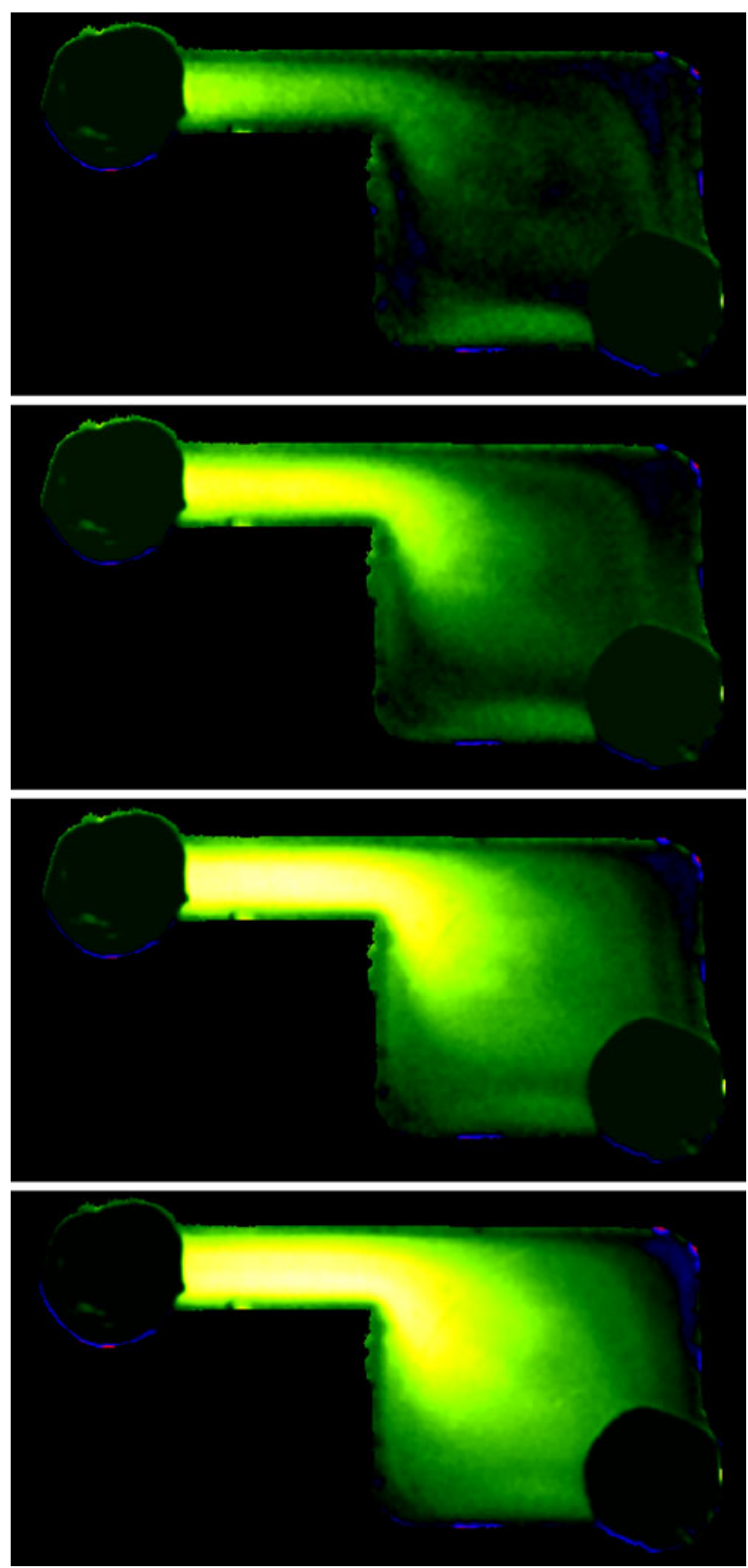

Fig. 10 Spatially resolved emission intensity of CCVJ in ethylene glycol flowing in the chamber depicted in Fig. 2b at 0.5, 0.75, 1 , and $1.5 \mathrm{ml} / \mathrm{min}$ (top to bottom). Emission intensity increases visibly with flow rate. Furthermore, the higher emission intensity in the narrow channel indicates a higher flow rate in the narrow channel compared to the wide basin

becomes particularly visible in the corresponding CFD simulation in Fig. 14: at $1 \mathrm{ml} / \mathrm{min}$ of flow, average simulated fluid velocity in the inflow channel was $1.1 \mathrm{~mm} / \mathrm{s}$, and in each of the two branches above and below the island, $0.53 \mathrm{~mm} / \mathrm{s}$. The regions that were considered for 

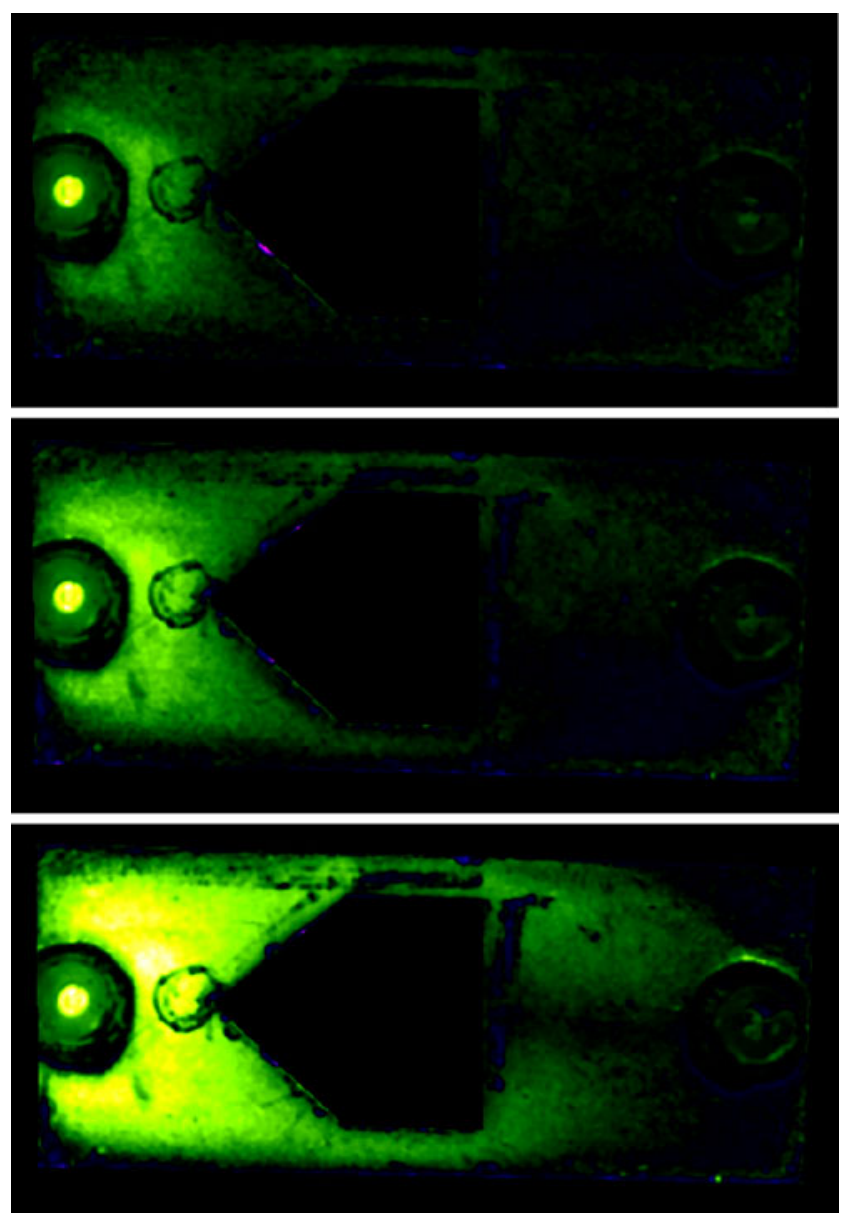

Fig. 11 Spatially resolved emission intensity of a third geometry at $0.5,1$ and $2 \mathrm{ml} / \mathrm{min}$ (top to bottom). Emission intensity increases visibly with flow rate, and particularly at high flow rates a dark line emerges to the right of the flow chamber where flow velocity is low because of the symmetry. Qualitatively, there is only a moderate relationship with the coputed fluid dynamics simulation in Fig. 12, and a noticeable reduction of intensity from left to right can be observed

averaging are labeled 1, 2a, and $2 \mathrm{~b}$ in Fig. 13. Average measured fluorescence intensity was determined inside these regions at several flow settings as shown in Fig. 15. At flow rates of $1 \mathrm{ml} / \mathrm{min}$ and above, measured

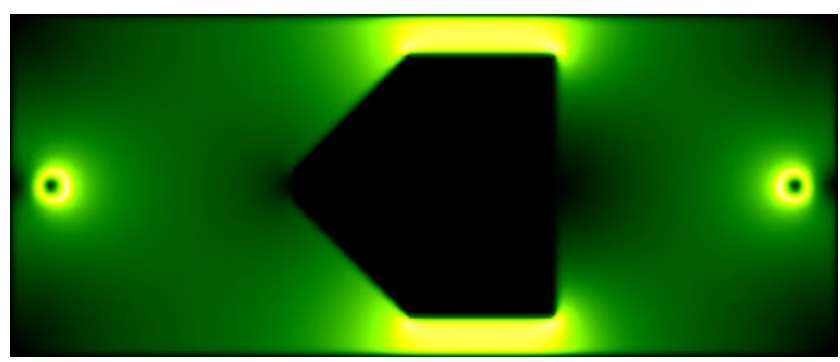

Fig. 12 Computed fluid dynamics simulation of flow in the chamber in Fig. 11 at $1 \mathrm{ml} / \mathrm{min}$
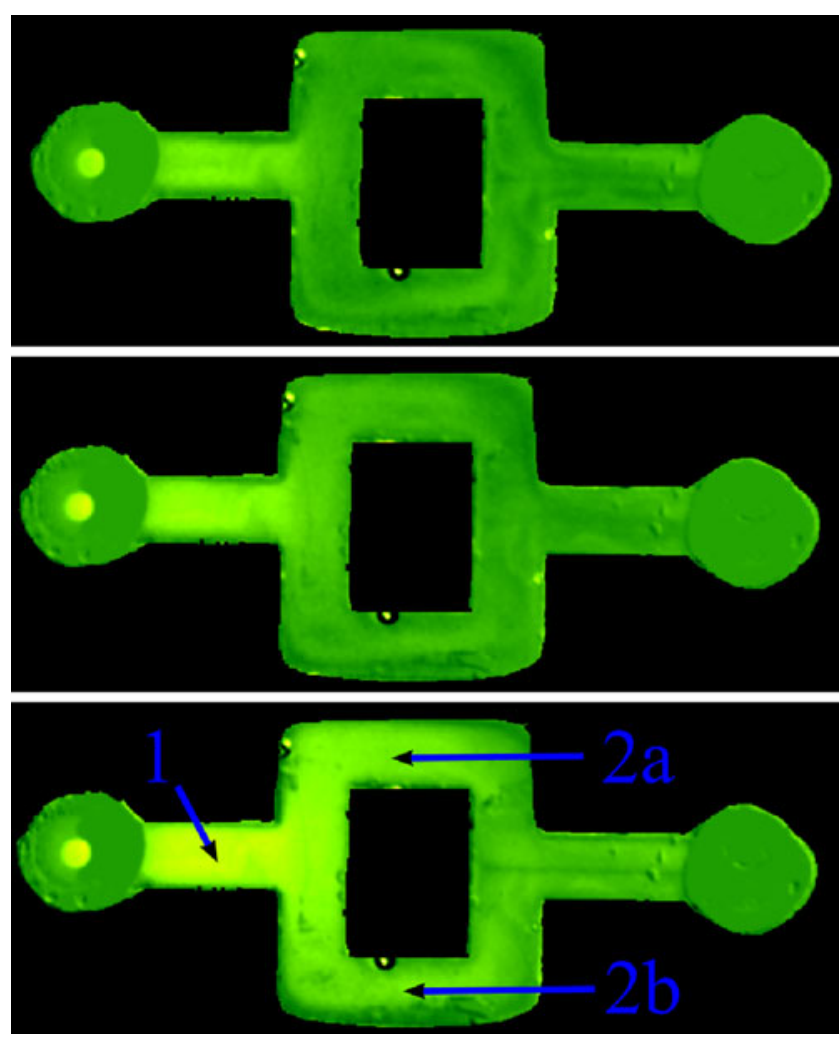

Fig. 13 Spatially resolved emission intensity of a fourth chamber geometry at $0.5,0.75$ and $1.0 \mathrm{ml} / \mathrm{min}$ (top to bottom). As the stream splits above and below the rectangular island, a decrease of the emission intensity (consistent with halving the flow rate) is seen. However, similar to the images in Figs. 4, 11 and 10, a fading of the intensity over the length of the channel is observed. For the analysis of average intensities, regions of interest $(1,2 \mathrm{a}$, and $2 \mathrm{~b}$ ) were defined

fluorescence intensity was about twice as high in the inflow channel (region 1) than in regions $2 \mathrm{a}$ and $2 \mathrm{~b}$. Because of the nonlinear nature of the flow-induced fluorescence increase (Eq. 4), this ratio increased towards lower flow rates where the fluorescence increase more closely approaches the detection limit in the upper and lower branches due to the lower velocity.

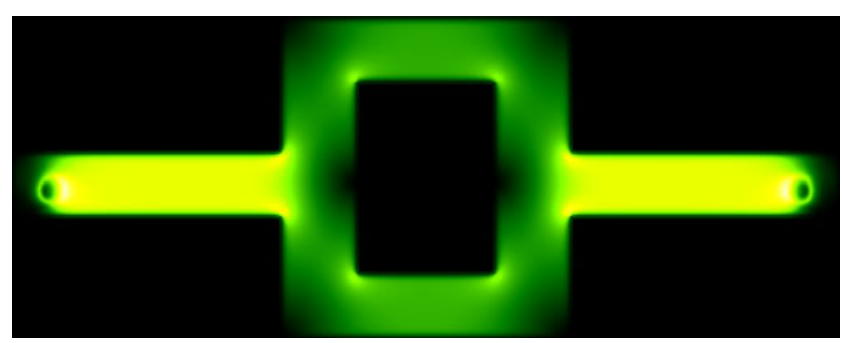

Fig. 14 Computed fluid dynamics simulation of flow in the chamber depicted in Fig. 13 at $1 \mathrm{ml} / \mathrm{min}$ 


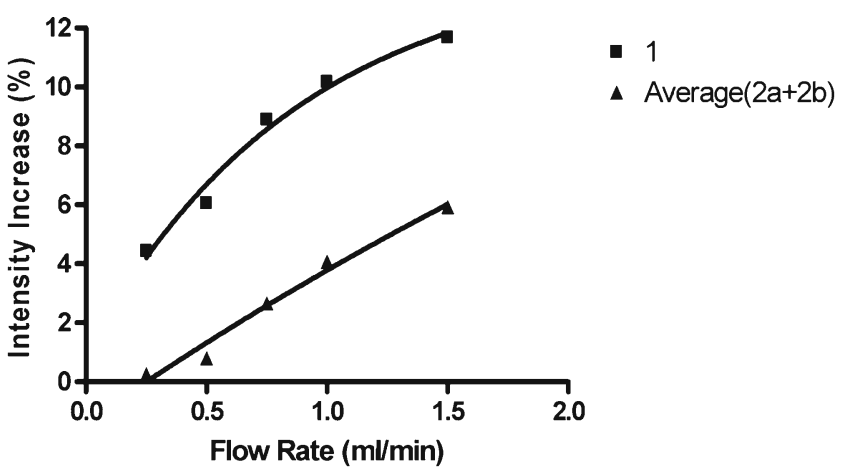

Fig. 15 Flow rate vs. emission intensity of CCVJ for region 1 and for the average of regions $2 \mathrm{a}$ and $2 \mathrm{~b}$ in the flow chamber from Fig. 13

\section{Discussion}

In this study, we demonstrated that molecular rotor solutions can be used to image flow patterns in fluidic chambers. The basic finding that molecular rotors show an increased fluorescence quantum yield in solvents was reported earlier [12], and for both dyes that were considered in this study, this effect was illustrated with the stirrer experiment in Fig. 3. In this study, we expanded on the original research by acquiring spatially resolved fluorescence, that is, fluorescence images.

The imaging method was based on low-cost equipment. A pair of collimated high-power LED provided excitation light very similar to laser induced fluorescence (LIF), but with the LED's advantage to illuminate larger areas more homogeneously. Image acquisition was performed with a consumer-grade SLR camera able to set a suitable exposure and integration time of up to $30 \mathrm{~s}$. The camera noise typically associated with higher sensitivity settings was eliminated by image processing: median filtering, image averaging, and subsequent Gaussian filtering. The major disadvantage of the camera was its propensity to perform its own internal image enhancement, which was primarily driven by the scene as perceived by the built-in camera software. Only after turning off all automatic functions, from automatic exposure to automatic white-balance, quantitative comparison between images became possible. Furthermore, the SLR camera acquires a color image. The highest intensity of the emitted fluorescence was found in the blue channel that had a peak sensitivity wavelength of $450 \mathrm{~nm}$. For this reason, the blue channel was extracted and used exclusively in the image processing steps. Addition of the green channel did not change the findings, and the red channel contained no image information.
Quantitatively, the flow-related intensity increase that was measured in the flow chambers followed the same exponential association that was found by fluorescence spectroscopy in simple flow tubes [12]. Notably, at high flow rates, a saturation effect was observed that can be described with Eq. 4. We hypothesized that the flow-sensitive effect is caused by polarpolar interaction between solvent and fluorophore [12]. Consistent with this notion, the more polar dye CCVJ showed higher sensitivity at low flow rates, but also more rapid saturation, than the less polar CPVDA dye. In addition, the maximum extrapolated intensity increase was about twice as high with CCVJ than with CPVDA. The solvent in this study was ethylene glycol, chosen for its relatively high viscosity that causes a relatively high no-flow emission intensity (Eq. 1). Different polar fluorophore/solvent combinations behave in a very similar manner, but the maximum intensity increase $\Delta I_{\max }$ (Eq. 4) is reduced in lowviscosity solvents. Although flow pattern imaging with the method described in this study is possible with, for example, CCVJ and water, sensitivity is lower, and random influences (noise) reduce the precision of the method. On the other hand, a monotonic nonlinearity in a sensor generally poses no problem as it can be calibrated with a suitable compensating calibration curve. In general, we anticipate that specially designed molecular rotors can provide an optimal response in a specific fluid [15]. Based on a recent study where modifications of the dipole led to strong wavelength shifts [15], we can envision molecular rotor solutions with nonoverlapping spectra being employed to examine binary fluids or mixing problems.

To obtain quantitative values for the fluorescence quantum yield, we performed steady-state spectroscopy with a reference fluorophore. At low dye concentrations, fluorescence quantum yield $\phi_{F}$ and emission intensity $I_{\mathrm{em}}$ are approximately related through Eq. 5,

$I_{\mathrm{em}}=\alpha \cdot c \cdot \phi_{F}$

where $\alpha$ is an instrument-dependent proportionality factor and $c$ is the dye concentration. At higher dye concentrations, the relationship between emission intensity and quantum yield follows an exponential function, and correction methods for high dye concentration are available [16]. With steady-state fluorescence spectroscopy, we compared the emission intensities of CCVJ and CPVDA to that of Lucifer Yellow under identical conditions, thus eliminating the instrument constant $\alpha$. Lucifer Yellow has a peak excitation and emission similar to CCVJ, and the quantum yield of Lucifer Yellow in ethylene glycol was found 
to be 0.53 [17]. We determined almost identical noflow values of $\phi_{F}=0.041 \pm 0.005$ for CCVJ and $\phi_{F}=$ $0.041 \pm 0.0025$ for CPVDA. The close agreement between the dye emission intensities, in spite of their different structure, was reported elsewhere [15]. With reference to Fig. 3, CCVJ increases its fluorescence quantum yield from 0.041 to 0.047 in the stirrer experiment, and CPVDA increases its fluorescence quantum yield from 0.041 to 0.043 .

We have employed CFD to qualitatively relate the flow pattern images to computed flow profiles. Since the fluorescence images are two-dimensional projections of the spatially resolved emission intensity, we also projected the three-dimensional CFD flow field onto a plane by averaging the velocity values parallel to the z-axis (direction of view). The best match between computer-simulated flow and fluorescence intensity was found in the second geometry (Figs. 2b, 9 and 10). A good match between CFD and fluorescence imaging was also found in the fourth geometry (Figs. 2d, 13, and 14). In the other two geometries, the fluorescence image was affected by an intensity fading effect that warrants closer inspection.

No effect of fading over time was observed in the stirrer experiment or in the fiber/tube experiments reported in our earlier study [12]. Possible explanations for the fading effect include photobleaching, local heating under bright LED illumination, and imperfections in the flow channel. The low average fluid velocity (in the widest channel, Fig. 2a, the fluid takes several minutes to cross the channel from left to right) makes photobleaching and heating likely candidates. Local heating would directly affect the emission intensity of a molecular rotor, because fluid viscosity generally decreases at higher temperatures. However, we were able to prove that neither photobleaching nor local heating account for the fading by taking multiple no-flow images at different time intervals. None of the no-flow images taken several minutes after the initial image showed a systematic reduction of the fluorescence intensity. We also measured stability of the LED excitation with a photodetector and found it accurate within below $1 \%$. The stability of the system over repeated exposures is demonstrated in Fig. 16, where ten successive acquisitions of the same flow chamber were taken in approximate one-minute intervals. The intensity shown is the averaged intensity over a region of interest encompassing the fluorescent area. No trend is visible that would indicate photobleaching or decreased intensity caused by local heating. Furthermore, we were able to exclude channel imperfections by reversing the flow direction and by examining the streamlines visualized by fluorescent beads (Fig. 8). By

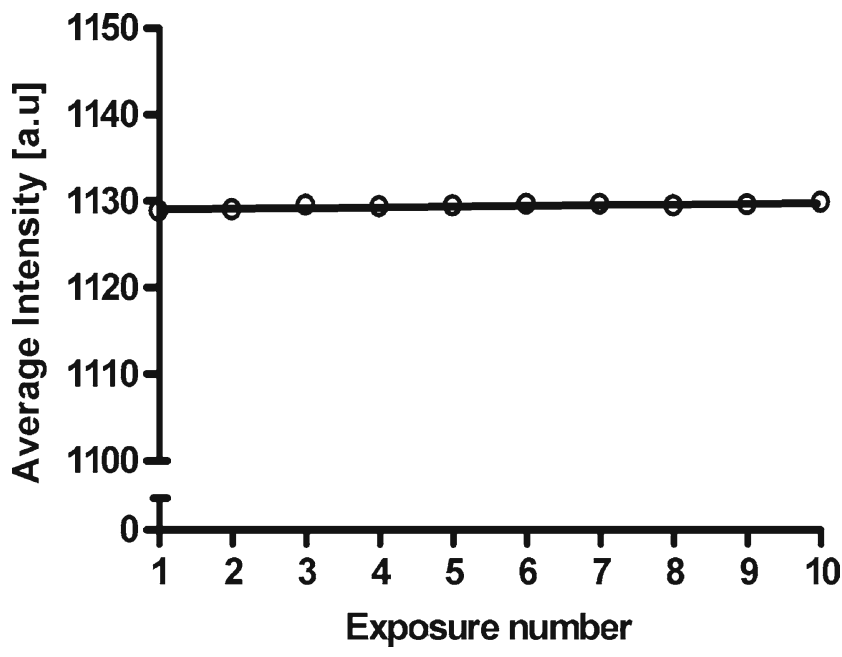

Fig. 16 Repeatability of the average fluorescent intensity of a flow chamber filled with fluorescent fluid under no-flow conditions. Ten successive acquisitions were taken in approximate oneminute intervals. The coefficient of variation is $0.03 \%$, and no trend towards higher or lower average intensity is discernible

reversing flow direction, we were also able to show that the solution recovers from the fading effect. In addition, we excluded unanticipated flow effects based on our CFD simulations (Fig. 7). Ester formation of the carboxylic acid CCVJ with the solvent alcohol could also be excluded, because the same fading effect was observed with the CPVDA dye, which cannot form ester bonds with alcohols. It is further conceivable that the dye noncovalently associates with the solvent under the influence of the excitation light. Although the exact mechanism of this association is still unknown, a light-induced mechanism is most likely to explain the fading effect. We partially occluded the basic chamber (Fig. 2a) and found diminished fading in the occluded areas (data not shown). In this context, it is interesting to note that the decay seems to be distance-dependent and not time-dependent. If we assume exponentialdecay behavior in Fig. 5, the decay constants were 6.3, $37.8,39.26$, and $42.77 \mathrm{~mm}$ for flow velocities of 0.14 , $0.21,0.28$, and $0.42 \mathrm{~mm} / \mathrm{s}$, respectively. With the exception of the low flow rate where the intensity increase rapidly drops to zero $(0.5 \mathrm{ml} / \mathrm{min}$, corresponding to $0.14 \mathrm{~mm} / \mathrm{s}$ ), the decay constants are very similar when expressed as distances, although the time it takes the fluid to travel over the distance of the decay constant varies from $102 \mathrm{~s}(1.5 \mathrm{ml} / \mathrm{min})$ to $180 \mathrm{~s}(0.75 \mathrm{ml} / \mathrm{min})$. If exposure to light was the main determining factor, the decay constant would vary with distance, but not with fluid velocity, in other words, the slower fluids should fade more rapidly than the faster fluids, which they don't. Finally, it appears qualitatively that wider flow channels (Figs. 4 and 11) exhibit more fading than 
narrower channels (Figs. 10 and 13). These observations nonwithstanding, the cause of the fading effect remains unknown.

A second question arises whether molecular rotors increase their intensity with flow velocity or with the shear rate or shear stress of the fluid. Intuitively, one would expect that the relative movement of the finite layers of solvent molecules, as described in the microviscosity model by Gierer and Wirtz [18], is the primary mechanism that influences the molecular rotor's reorientation rate. In fact, we concluded from earlier experiments that emission intensity would increase with fluid shear stress rather than with fluid velocity [12]. However, the observations in this study point at an intensity increase driven by flow rather than by shear stress. Figure 11 supports this notion with the flow profile perpendicular to the flow direction in the narrow sections above and below the island. Computation of the shear rate profile from Fig. 12 indicates that the shear rate profile has a distinct minimum in the center of the channel. Conversely, the velocity profile shows the highest velocity in the center, with the velocity tapering off towards the chamber walls. The second profile is matched by the intensity profile in Fig. 11. To further demonstrate this point, we computed not only the projected flow pattern in Fig. 9, but also the projected shear rate from the velocity field according to Eq. 6:

$|\tau|=\sqrt{\left(\frac{\partial v_{x}}{\partial x}\right)^{2}+\left(\frac{\partial v_{y}}{\partial y}\right)^{2}+\left(\frac{\partial v_{z}}{\partial z}\right)^{2}}$

The resulting shear pattern (Fig. 9, bottom) is relatively complex with a prominent symmetry line running diagonally across the basin, and with relatively low shear in the inflow region that is characterized by very homogeneous flow. The qualitative comparison of computed velocity and shear pattern with the fluorescent images provides strong evidence that the fluorescent pattern correlates to flow velocity rather than shear. The question whether fluid velocity or shear stress causes the intensity increase is fundamental to the understanding of the underlying mechanism of shear stress sensitivity. Therefore, this question needs to be illuminated with additional experiments, with geometries designed to maximize the difference between flow and shear stress patterns, and with fluids of different viscosity.

These open questions non-withstanding, the experiments in this study give rise to an interesting and unique application of molecular rotors. Flow patterns can be imaged at extremely high resolutions (limited only by the resolution of the optical apparatus) and in real time (limited by the acquisition speed of the optical apparatus). Intensity changes were observed with average flow velocities as low as $0.1 \mathrm{~mm} / \mathrm{s}$. This represents an extremely high sensitivity for a flow measurement system. This sensitivity can be matched by particle tracking systems, but we expect to exceed both temporal and spatial resolution of particle tracking as our methods matures. Out of several mainstream velocity measurement methods (such as Doppler ultrasound), only flow meters that are based on a heated plate can match the sensitivity of our methods. In these flow meters, the heated plate gets cooled by the fluid flowing over the plate, and temperature measurement provides velocity. However, such a system cannot match the molecular rotor's spatial resolution.

Therefore, molecular rotors promise to provide an alternative flow measurement method, because they represent a new generation of extremely sensitive flow sensors with low-cost instrumentation. Potential applications can be found in the fields of microand nano- fludics, including mixing optimizations, flow measurement, closed loop flow control, flow and shear imaging, and the validation of computed fluid dynamics models.

Acknowledgements The authors gratefully acknowledge research support from the NSF through grant CMMI-0652476 and partial support through NIH grant 1R21 RR 025358.

Open Access This article is distributed under the terms of the Creative Commons Attribution Noncommercial License which permits any noncommercial use, distribution, and reproduction in any medium, provided the original author(s) and source are credited.

\section{References}

1. Haidekker MA, Theodorakis EA (2007) Molecular rotors fluorescent biosensors for viscosity and flow. Org Biomol Chem 5(11):1669-1678

2. Loutfy RO (1986) Fluorescence probes for polymer freevolume. NATO ASI Ser Ser C Math Phys Sci 182:429-448

3. Loutfy RO, Arnold BA (1982) Effect of viscosity and temperature on torsional relaxation of molecular rotors. J Phys Chem 86(21):4205-4211

4. Kung CE, Reed JK (1986) Microviscosity measurements of phospholipid bilayers using fluorescent dyes that undergo torsional relaxation. Biochemistry 25(20):6114-6121

5. Haidekker MA, Tsai AG, Brady T, Stevens HY, Frangos JA, Theodorakis E et al (2002) A novel approach to blood plasma viscosity measurement using fluorescent molecular rotors. Am J Physiol 282(5):H1609-H1614

6. Akers WJ, Haidekker MA (2005) Precision assessment of biofluid viscosity measurements using molecular rotors. J Biomech Eng 127:450-454

7. Allen BD, Benniston AC, Harriman A, Rostron SA, Yu C (2005) The photophysical properties of a julolidene-based molecular rotor. Phys Chem Chem Phys 7(16):3035-3040 
8. Grabowski ZR, Rotkiewicz K, Rettig W (2003) Structural changes accompanying intramolecular electron transfer: focus on twisted intramolecular charge-transfer states and structures. Chem Rev Columbus 103(10):3899-4032

9. Förster T, Hoffmann G (1971) Die Viskositätsabhngigkeit der Fluoreszenzquantenausbeuten einiger Farbstoffsysteme (Effect of viscosity on the fluorescence quantum yield of some dye systems). Z Phys Chem 75:63-76

10. Loutfy RO, Law KY (1980) Electrochemistry and spectroscopy of intramolecular charge-transfer complexes. pN, Ndialkylaminobenzylidenemalonitriles. J Phys Chem 84:28032808

11. Haidekker MA, Brady TP, Chalian SH, Akers W, Lichlyter D, Theodorakis EA (2004) Hydrophilic molecular rotor derivatives-synthesis and characterization. Bioorganic Chem 32(4):274-289

12. Haidekker MA, Akers W, Lichlyter D, Brady TP, Theodorakis EA (2005) Sensing of flow and shear stress using fluorescent molecular rotors. Sens Lett 3:42-48
13. Haidekker MA (2010) Advanced biomedical image analysis. Wiley, New York, ISBN 978-0-470-62458-6 (in press)

14. Griebel M, Dornseifer T, Neunhoeffer T (1998) Numerical simulation in fluid dynamics: a practical introduction. Society for Industrial and Applied Mathematics, Philadelphia, PA, USA

15. Sutharsan J, Lichlyter D, Wright NE, Dakanali M, Haidekker MA, Theodorakis EA (2010) Molecular rotors: synthesis and evaluation as viscosity sensors. Tetrahedron 66:2582-2588

16. Nighswander-Rempel S, Riesz J, Gilmore J, Bothma J, Meredith P 2005) Quantitative fluorescence excitation spectra for synthetic eumelanin. J Phys Chem B 109(43):20629_ 20635

17. Nalin CM, Snyder B, McCarty RE (1985) Selective modification of an $\alpha$ subunit of chloroplast coupling factor 1. Biochemistry 24(9):2318-2324

18. Gierer A, Wirtz K (1953) Molekulare theorie der mikroreibung (Molecular theory of microfriction). Z Naturforsch 8:532-538 